\title{
A Multi-Agent Approach to Design a WLAN System
}

\author{
https://doi.org/10.3991/ijoe.v13i11.7757 \\ You Zheng \\ Wuhan University of Technology, Wuhan, China \\ Xiaohuo $\mathrm{Xu}^{(凶)}$, Tailong Shi \\ Ningbo University of Technology, Ningbo, China \\ huosai008@qq.com \\ Dandan Fang \\ Wuhan University of Technology, Wuhan, China \\ Peng An \\ Ningbo University of Technology, Ningbo, China
}

\begin{abstract}
There have been a number of research works that investigate the use of formal optimization techniques for the accurate design of WLAN. Unfortunately, these approaches may not be effectively applied to a large scale WLAN deployment scenario. Consequently, it is necessary to design an algorithm that has more powerful ability of global optimization to solve WLAN deployment problems. The main contribution of the work presented in this paper is designing a multi-agent optimization algorithm based on the local control from agents and global control from system, including the synthetical analysis of the relationship of channel and field strength in WLAN. The results of experiments indicate that our multi-agent optimization algorithm is much more efficient comparing with the present agent-based algorithm for the practical engineering project with low density candidate sites.
\end{abstract}

Keywords - WLAN planning, multi-agent, optimization, large scale WLAN

\section{Introduction}

With the growing commercial interest in mobile Internet, telecom operators are keen on constructing the infrastructure of hotspot. Among many wireless communication technologies, WLAN has been the best choice because of its advantages, such as low cost for network installation and maintenance, using free frequency band, mature network technology, numerous intelligent terminals used. The QoS(Quality of Service) is most commonly used criterion to estimate the reasonability of a WLAN deployment. With the number of access points(APs) increasing, the capacity of WLAN coverage will increase, whereas the QoS might be worse due to the limitation of the legally allowed frequency band, and the installation cost will rise. These are major 
drawbacks. To search for a suitable deployment of APs is so-called WLAN network planning problem.

Some studies have been proposed in the design of WLAN network planning. Integer programming and quadratic programming were applied in some researches [1][2]. However, their performance will decline sharply once confronted with a large scale WLAN planning problem. Some researchers put forward using metaheuristic algorithms such as Genetic Algorithm(GA) [3], Variable Neighborhood Search(VNS) [4] to find the best design. Metaheuristic algorithms often use the information in the past search process to find the next search direction. Some works prove that the metaheuristic algorithms are effective in cellular network planning [5][6]. But in indoor environment the interferences of obstacles make the radio propagation become more and more complex, which makes the solution space of the deployment problem extremely complicated. And it is also hard to make sure the search directions of heuristic algorithms.

The variable neighborhood search algorithm [7] is a kind of trajectory metaheuristics. This algorithm mainly focuses on the improvement of the quality of one solution, weakening the interaction and cooperation between different solutions, which may result in poor efficiency. Population-based metaheuristics such as genetic algorithm [8] usually emphasize on the overall efficiency at the expense of individual in-depth search. In order to avoid the defect of them, a new tool based on multi-agent is put forward in this paper to plan the WLAN network. In recent years, optimization technology based on multi-agent has been widely used in various problems [9][10][11] and obtained remarkable results. Multi-agent intelligent algorithm is a dynamic collaboration model, which based on the interactions and changes of relationships between different agents. The performance of an agent depends on not only itself, but also the behaviors of other agents. The objective of systematic learning is to find a strategy to maximize its own utility and the performance of the whole swarm of agents in the future.

In Alan Mc Gibney's researches [12][13], an agent-based optimization algorithm using the tool of game theory [14] was come up with. Although this algorithm is qualified to solve some large scale WLAN planning problems, it is not suitable to be applied to complex WLAN network planning problems. Because his researches do not take into account the relationship between the AP location and AP frequency, which makes the optimization algorithm ignore the good parts in solution space. Despite his paper using a growing neural gas network [15] which can generate high density candidate sites to reduce the complexity of solution space and weaken the integrated relationship between AP location and AP frequency in solution space, many WLAN planning cases in practical engineering have to design the candidate sites where APs may be installed under different circumstances including low density candidate sites that are defined in advance. That means his algorithm is limited to some large scale WLAN planning problems whose solution space may be simple. In addition, AP configurations include not only AP location and AP frequency mentioned in [12], but also AP azimuth and AP power, which are also important in WLAN design. Therefore, it is necessary to consider them in agent-based optimization algorithm, which is one of the improvements in this paper. 
Aiming at the WLAN deployment problem with low-density candidate sites and complicated optimization solution space, it is proposed that a multi-agent algorithm needs to be combined with the analysis of the internal relationship between location, power, azimuth, and frequency of an AP. In the algorithm, the relationship between frequency and field strength is taken into account and overall control from a system consisting of a group of agents is introduced to increase the possibility to escape from the local optimal solutions.

The rest of the paper is organized as follows. The next section describes the definition of WLAN communication model and optimization problem. In Section 3 the design of improved multi-agent will be proposed. The experiments and results are given in section 4. Final section gives the conclusion and perspective of this paper.

\section{WLAN Mathematical Model and Optimization Problem}

Research on WLAN design has been carrying out for decades. Some mathematical models about WLAN deployment can be studied from some literatures [16][17]. In this paper, to facilitate the handling of problem, the WLAN optimization model is decomposed into some sub-models:

1. AP Model, where a finite set of candidate sites and candidate APs are predefined and the parameters setting of each AP type such as azimuth, emitted power and frequency channel are predefined;

2. Radio Signal Model, where the signal strength for wireless communication in the building is predefined;

3. User Requirement Model, where the bit rates required by users are predefined;

4. Throughput Model, where the evaluation of real bit rates as well as the association rules between the clients and the AP are predefined.

The purpose of these sub-models is to define the variables for WLAN optimization problem so as to obtain the decision variables and objectives of the optimization problem. The internal linkages among these models are represented in Figure 1 and then the models are described in the next section.

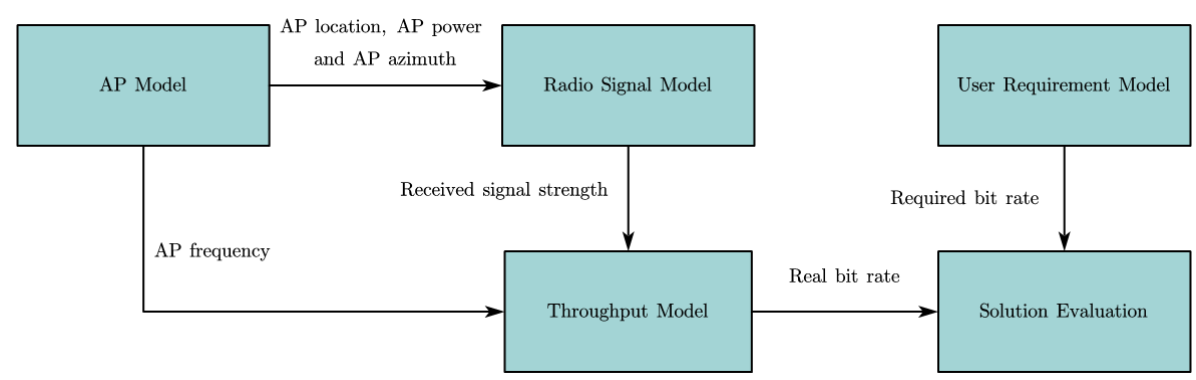

Fig. 1. The internal linkages among the sub-models for optimization 


\subsection{WLAN model}

AP Model. In this model, candidate site locations and AP configurations are defined. A candidate site is a geographical location where an AP can be deployed. When an AP is installed on a candidate site, its configurations including channel, azimuth, and emitted power are supposed to be set up. In EU and China, there are 13 available channels under the $802.11 \mathrm{~b} / \mathrm{g}$ standard, which are concentrated in the frequency band of 2.4G. The azimuth is an angular measurement in spherical coordinate system, which is defined as the angle between the North and the antenna radiation direction in the horizontal plane in our WLAN model. The emitted power is the power of the transmitter, and its units are in $\mathrm{dBm}$. The emitted power represents the radiation ability of AP. The higher emitted power can bring about the increase in the range of AP radiation, but the more serious channel interference with nearby APs would occur. Therefore, it is necessary to limit the magnitude of the emitted power.

Mathematically, some notations and definitions are described as follows:

- The set of candidate sites for WLAN design is denoted by $S=\left\{s_{1}, \ldots, s_{i}, \ldots, s_{n s}\right\}$. Here, $n s$ is the number of candidate sites, and $s_{i}$ is the $i^{\text {th }}$ candidate site.

- The set of empty candidate sites is defined by $S^{e}=\left\{s_{1}^{e}, \ldots, s_{i}^{e}, \ldots, s_{n e}^{e}\right\}$.Here, ne is the the number of empty candidate sites and $s_{i}^{e}$ is the $i^{t h}$ candidate site unoccupied by an AP.

- The set of AP locations is denoted by $L=\left\{l_{1}, \ldots, l_{i}, \ldots, l_{n l}\right\}$. Here, $n l$ and $l_{i}$ represent the number of candidate sites with installed APs and the $i^{\text {th }}$ candidate site occupied by an AP, respectively. Obviously, $S=L \cup S^{e}$.

- The set of available channels is denoted by $F=\left\{f_{1}, \ldots, f_{i} \ldots f_{n F}\right\}$. Here, $n F$ is the number of available channels, and $f_{i}$ is the $i^{\text {th }}$ channel.

- The set of the azimuths is denoted by $H$, which is predefined for different antenna types. For example:

$\mathrm{H}=\{\overrightarrow{0}\}$ for omni-directional antennas.

$\mathrm{H}=\left\{0^{\circ}, 45^{\circ}, 90^{\circ}, 135^{\circ}\right\}$ for bi-directional antennas.

$\mathrm{H}=\left\{0^{\circ}, 45^{\circ}, 90^{\circ}, 135^{\circ}, 180^{\circ}, 225^{\circ}, 270^{\circ}, 315^{\circ}\right\}$ for other directive antennas.

Here, the size of $H$ is denoted by $n H$.

- The set of different levels of emitted powers is denoted by $P$. The elements of $P$ are determined by the engineering rules of manufacturers. Here, the size of $P$ is denoted by $n P$.

Then, one AP can be characterized by the following variables $(l, p, h, f)$. Here, $l$ is denoted by the candidate site where the AP is installed, $p$ is its emitted power, $h$ is its azimuth, and $f$ is its channel.

Radio Signal Model. To achieve a communication service, a user terminal should receive enough signal strength from an AP. If the signal strength received by a user terminal is greater than receiver sensitivity, the user will be covered by at least one AP. In most cases, a user is covered by several APs, but its communicating terminal only connects with serving AP which usually offers the best signal strength for it.

For ease of calculation of received signal strength(RSS), the continuous space in real world is discretized into a grid of pixels because the WLAN mathematical model 
is a discrete model that is suitable for computer simulation and calculation. The pixel center, called Marking Point(MP), is used to denote the whole pixel.

Mathematically, some notations and definitions are described as follows:

- The minimum RSS that a user terminal can sense is denoted by rss $^{\min }$. Obviously, a user whose all mean RSSs are smaller than $r s s^{\min }$ does not connect any AP.

- The mean RSS in MP $m$ and coming from the AP whose location is $l$ is denoted by $r s s_{m}^{l}$.

With the AP characteristic $(l, p, h, f)$, the RSS expression at MP $m$ is expressed by $r s s_{m}^{l}=\chi(m, l, p, h, f)$. Here, $\chi($.$) is a function related to the indoor propagation model and$ the units are in $\mathrm{dBm}$.

- The set of the RSSs at MP $m$ which are from all installed APs is denoted by $R S S_{m}=\left\{r s s_{m}^{l} \mid \forall l \in L\right\}$.

User Requirement Model. In order to define the user demand, communication zone where users desire for wireless communication is defined. The MP unoccupied by any AP in the communication zone is called Test Point(TP) where People have requirements for communication speed.

Mathematically, some notations and definitions are described as follows:

- The set of mean RSSs at TP $t$ which come from all installed APs and are beyond $r s s^{\min }$ is denoted by $R S S_{t}^{b}=\left\{r s s \mid m=t, r s s \in R S S_{m}, r s s \geq r s s^{\min }\right\}$.

- The number of users at TP $t$ is denoted by $n_{t}^{u}$.

- The bit rate of a user requirement is denoted by $r^{u}$.

Throughput Model. In this model, the main task is to acquire the real bit rate offered to the users. A wise way to model the throughput is to use the signal to interference plus noise ratio (SINR). In fact, SINR only determines the user's nominal bit rate, and the user's real bit rate is smaller than its nominal rate due to the additional overhead such as protocol overhead, switching to ensure compatibility and idle guard times built into the protocol. The real bit rate which a user can gain from its serving AP is primarily determined by the number of users connecting to this AP and their SINRs. When nominal bit rate and MAC scheme are determined, the relevant computation method that is used to obtain the total throughput provided by an AP can be found in [18].

Mathematically, some notations and definitions are described as follows:

- The total throughput offered to all users at TP $t$ is denoted by $r_{t}^{T}$.

- The real bit rate of one user at TP $t$ is denoted by $r_{u}^{t}$.

In this paper, it is assumed that channel access is shared equitably among the clients. In other words, an AP sends the same number of frames to all its customers. Then, the real bit for a user in TP $t$ can be expressed by $r_{u}^{t}=r_{t}^{T} / n_{t}^{u}$. 


\subsection{Optimization problem}

After building the sub-models, the optimization problem is established. In this section, decision variables, optimization objectives and constraint conditions will be given.

Decision variables. A candidate site configuration is selected as a decision variable because the solution space is determined by the distribution density of the candidate sites. The solution of the problem is defined as $x=\left(x_{1}, \ldots, x_{i}, \ldots, x_{n x}\right)$. Here, $x_{i}$ represents the $i^{t h}$ configuration of candidate site and $n x=n s . x_{i}=\Phi$ if the $i^{\text {th }}$ candidate site is unoccupied by any AP. $x_{i}=(p, h, f)$ if an installed AP is on the candidate site $s_{i}$ and its channel frequency, azimuth, and emitted power are $f, h, p$, respectively.

Optimization objectives and constraint conditions. The ultimate goal of a WLAN design is to maximize the user satisfaction of communication service. At the same time, the less the APs are, the lower the cost for deployment in WLAN design is. Therefore, the total lack of QoS and the number of APs are used as the optimization objectives of a solution, which is a multi-objective optimization problem in mathematics. There are three methods that can deal with multi-objective optimization: the first is a weighting method, the second is a ranking method, and the third is an epsilon - constraint method [19].

The key of ranking method is making the secondary objective reach the optimal value as far as possible when under the condition that main objective has reached minimum (maximum). For the reason that the satisfaction of network users is the primary consideration and the WLAN deployment cost is a secondary consideration in WLAN problems, ranking method is applied to dispose the WLAN optimization problem in this paper. The descriptions of the optimization problem are as follows:

$$
\text { To minimize }: o b j=\sum_{t \in t_{c}} \max \left(n_{t}^{u} \times r^{u}-r_{t}^{T}, 0\right)
$$

Here, $T_{c}$ is the set of TPs in communication zone. obj is the total lack of QoS, which is a main objective of current WLAN configuration. Obviously, the smaller $o b j$ is, the better WLAN plans.

Constraint 1: The number of installed APs does not exceed the maximum allowed number of APs.

This constraint can be expressed as $n l \leq n l_{\max }$, Here, $n l_{\max }$ represents the maximum allowed number of APs.

Constraint 2: All the decision variables of the problem should be included in their domain of definition.

This constraint can be expressed as $x_{i}=\Phi$ or $x_{i}=(p, h, f), p \in P, h \in H, f \in F$.

Constraint 3: In the case of main objective obj achieving optimal value, $n l$ is made to reach minimum as far as possible, which is a secondary objective of current WLAN deployment.

If there exist two WLAN configurations whose main objectives are the same, then their AP numbers will be compared. The WLAN configuration with less number of APs has higher evaluation for its saving cost for AP installation. 


\section{Improved Agent-Based Optimization Algorithm}

In this section, an improved agent optimization algorithm is designed. An AP is regarded as an agent, and all APs in communication zone form a distributed multi-agent system. These agents can sense users who connect to them. They can follow the common rules to take actions, and predict the change of the total lack of QoS before an action is carried out. Owing to one agent has no ability of global control without which the algorithm is hard to jump out of local optima, the global control should be made by the multi-agent system itself so as to increase the range of search in solution space. The global control includes adding an agent and deleting an agent.

\subsection{Multi-agent system description}

Agent percepts. Agent percepts are the premise of agent making reasonable decision. The agent percepts for WLAN design include position, channel, azimuth, emitted power, utility function and the users who connect to it.

Users in the region are divided into two categories: one can receive wireless signal, and the other is unable to connect any agent because the received signal cannot be sensed by the mobile terminal. It is assumed that user terminal only connects to the agent which is sensed and provides maximum wireless signal strength to it. Therefore, an agent can only perceive the users who can communicate with it.

Agent can perceive its position on a candidate site. The agent can leave the original position to move to another candidate site location that is not occupied by any other agent. At the same time, the agent can perceive its own channel, azimuth, emitted power. Channel determines the frequency relation among agents. The local field strength distribution around the agent is influenced by azimuth and emitted power.

The purpose of an agent is to satisfy the total bit rate requirements of users who connect to it as far as possible, so the utility function can be expressed as the sum of the bit rates that agent offers. Mathematically:

$$
u_{i}=\sum_{t \in T_{\text {agent }}} n_{t}^{u} \times \min \left(r^{u}, r_{u}^{t}\right)
$$

Here, $u_{i}$ is a utility function of the $i^{\text {th }}$ agent. $T_{\text {agent }}$ represents the set of the TPs where users connect to the $i^{\text {th }}$ agent.

Cooperative game. An agent taking actions from its own view may reduce other agents' utility function values and increase the total lack of QoS in communication zone, which leads to the optimization algorithm search towards the direction of bad solution. So, it is necessary to restrict actions of agents by the tool of cooperative game [20]. In cooperative game, an agent can take only one action in its turn and the next agent cannot take any action until the last agent ends the game. What's more, in order to ensure the high QoS of WLAN, actions of an agent will be limited until it forecasts that the QoS will be better after taking one action. An action taken by an agent which leads to worse QoS is regarded as an irrational behavior. 
Action rules. The search direction of algorithm in solution space depends on actions of agents. Rational actions could ensure high efficiency of algorithm and get rid of premature convergence. If an agent takes an action, at least one parameter will be changed. The rationality of an action depends on the relationships among agents, which are based on channel and field strength distribution.

As a matter of fact, when agents' frequencies are in the same frequency band, changing the channel of an agent does not affect the field strength distribution in communication zone. If it adjusts its channel, only the relationship of channel interference between the agent and other nearby agents will be changed.

In most cases, an agent moving to another new candidate site unoccupied by an AP will bring about serious frequency interference to agents around the new candidate site, which makes the good frequency relationships among the agents broken. Besides, the field strength distribution of the old position and new position may be awful when it moves. Due to the reasons above, the move operation is often limited in the cooperation game, which leads to the poor performance of multi-agent system. In order to decrease the restrictions of an agent and avoid the bad direction of search, it is necessary to perform frequency operation after moving new place. In this paper, the compound action for moving and frequency operation is deemed to one action.

Because both azimuth and emitted power of an agent only affect the local field strength around it, these two parameters are comprehensive consideration and three kinds of manners of compound action can be selected: 1 . Adjusting the azimuth, when the emitted power remains unchanged; 2 . The azimuth remains invariability, while the emitted power change; 3 . The azimuth and the emitted power change at the same time. Apart from that, to reduce the frequency interference, it is necessary for an agent to adjust the channel after the integrated adjustment of the azimuth and emitted power. Accordingly, the compound action of azimuth adjusted, changing power and frequency operation is also regarded as one action in this paper.

Global control. Different numbers of agents correspond to different parts of the solution space. A fixed number of agents will lead to the limited range of solution space for algorithm optimization. As a result, multi-agent should reasonably adjust the number of agents, which includes adding an agent and deleting it.

In our WLAN model, the maximum bit rate of an agent is $5500 \mathrm{~kb} / \mathrm{s}$, which means the total output of current agent group is finite. Besides, the covering capacity of an agent is also limited. Therefore, it is necessary to generate a new agent in multi-agent system. When all actions of agents are restricted in cooperative game, a temporary agent may be generated in current multi-agent system to improve QoS and jump into another part of solution space. The azimuth and emitted power of temporary agent is random. Once the temporary agent is created, it will find the best combination of channel and position for maximizing utility. After finding that combination of channel and position, the total lack of QoS will be forecasted. If the predicted value decreases, the temporary agent will join in current system. Otherwise, the temporary agent will have to seek the new combination of channel and position which only focuses on minimizing the total lack of QoS by greedy strategy. Then, it will predict the total lack of QoS again. If both the old and new predicted value are no reduction, the temporary agent will disappear. 
Once the solution space of problem is complex, the redundant agents may occur in current system, which bring more harms to the system than benefits. To reduce the number of useless agents, the system will delete the agent that has the worst performance among the redundant agents when all agents' actions are limited. If the agent is successfully deleted, the generation of new agent will not be taken into consideration temporarily until no redundant agent can be found and all agent's action are restricted in current system.

\subsection{Modified Agent-Based Optimization Algorithm}

Improved multi-agent algorithm consists of two sections. One describes cooperative game among agents in a group. Only one agent is allowed to take an action for maximizing its own utility in each iteration of cooperation game. Before taking an action, the agent will forecast the change of the total lack of QoS. If predicted value is bad, the agent will be restricted. The other section describes the regulatory mechanism of multi-agent system, which optimizes WLAN from global view. The regulatory mechanism is addition or deletion of an agent.

The section of cooperative game. In this section, three possible actions can be selected by agents. These are only changing channel (Only_Channel), moving and adjusting channel (Move_Channel), or adjusting azimuth, power and channel (Az Po Channel). To demonstrate the processes of these actions, let $\operatorname{Action}\left(i, t_{a}\right)$ be a function of the actions of the $i^{\text {th }}$ agent. $t_{a}$ has three possible values that correspond to different types of actions. Their specific relationships and the pseudo code of function $\operatorname{Action}\left(i, t_{a}\right)$ are shown in Table 1 and Algorithm 1 , respectively.

Table 1. The corresponding relationship between $t_{a}$ and the type of action

\begin{tabular}{|c|c|}
\hline $\mathbf{t}_{\mathbf{a}}$ & The type of action \\
\hline 0 & Only_Channel \\
\hline 1 & Az_Po_Channel \\
\hline 2 & Move_Channel \\
\hline
\end{tabular}

In Algorithm 1, if agent $i$ take an action successfully, it will return 1. Otherwise it will return 0. Some notations and definitions in Algorithm 1 are defined as follows:

- $c_{i}^{a}$ is the configuration of agent $i$, and $c_{i}^{a}=\left(s_{i}^{a}, p_{i}^{a}, h_{i}^{a}, f_{i}^{a}\right)$. Here, for agent $i, s_{i}^{a}, p_{i}^{a}, h_{i}^{a}$, $f_{i}^{a}$ are its site position, emitted power, azimuth and frequency, respectively.

- $U_{i}(c)$ represents the utility achieved by agent $i$ if its configuration is $c$.

- $\operatorname{Obj}(i, c)$ represents the total lack of QoS which is calculated under the condition that the configuration of agent $i$ is $c$ and other agents' configurations remain unchanged.

Algorithm 1. The function of action

$\operatorname{Action}\left(i, t_{a}\right)$ :

1. If $t_{a}$ is equal to 0

2. $C=\left\{\left(s_{i}^{a}, p_{i}^{a}, h_{i}^{a}, f\right)|f \in F|\left\{f_{i}^{a}\right\}\right\}$ 


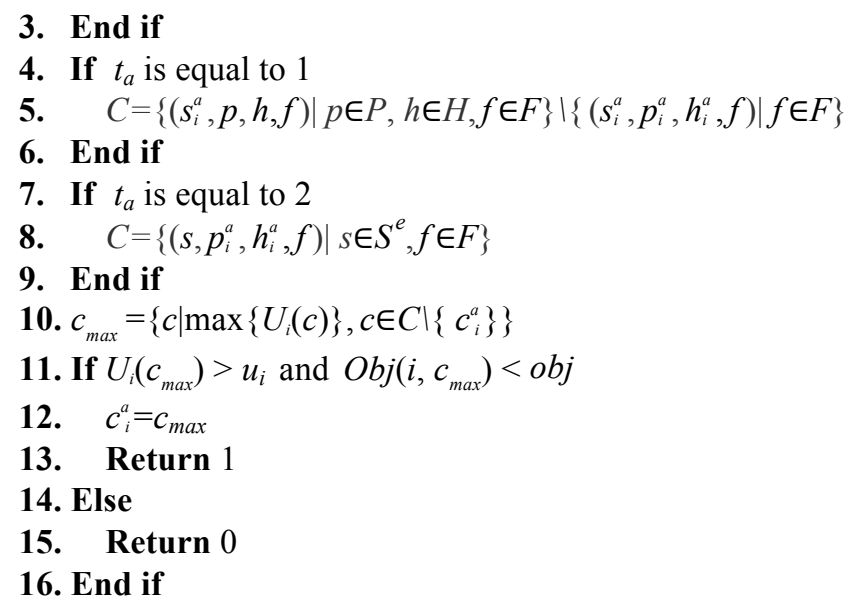

In the Algorithm mentioned above, $C$ is a set of the neighbor configurations of agent $i$, which is related to the type of action. Table 2 shows the relationship between the set $C$ and the type of action.

Table 2. The relationship between the size of set $C$ and the type of action

\begin{tabular}{|c|c|c|}
\hline The type of action & $\begin{array}{c}\text { Different parameter(s) among the neigh- } \\
\text { bor configurations in } \boldsymbol{C}\end{array}$ & The size of set $\boldsymbol{C}$ \\
\hline Only_Channel & Frequency & $n F-1$ \\
\hline Az_Po_Channel & Azimuth, power, frequency & $(n H \times n P-1) \times n F$ \\
\hline Move_Channel & Position, frequency & $n e^{\times} n F$ \\
\hline
\end{tabular}

In the actual project, for the reason that the size of set $C$ generally reaches the maximum value if the type of action is Move_Channel and Move_Channel has greatest impact on the field strength distribution in communication zone, Move_Channel is finally considered. In multi-agent system, Only_Channel is the top priority action because it does not change the relationship of field strength among agents. What's more, the size of its corresponding set $C$ is smallest, which consumes the least amount of computing time. Therefore, the process of cooperative takes Only_Channel first, and Move_Channel is considered finally if Only_Channel and AzPo_Channel are limited. Algorithm 2 demonstrates the process of cooperative game for agent $i$. The output of this algorithm is related to the configuration of agent $i$. If its configuration changes, the algorithm will return 1 finally. Otherwise it will return 0.

Algorithm 2. The process of cooperative game

Cooperative_game $(i)$

1. $m=0$

2. $m=\operatorname{Action}(i, 0)$

3. If $m$ is equal to 0

4. $m=\operatorname{Action}(i, 1)$ 


\section{End if \\ 6. If $m$ is equal to 0 \\ 7. $m=\operatorname{Action}(i, 2)$ \\ 8. End if \\ 9. Return $m$}

The section of global control. This section demonstrates the system control the number of agents from a global perspective. Algorithm 3 describes this process. Addition of an agent can increase the total throughput of the network when the number of agents is relatively small. However, the system composed of agents exists a redundant agent sometimes. Therefore, deleting an agent is firstly considered (from line 2 to line 6 ), and addition of an agent will be considered if deleting one agent fails. According to the different purposes, addition of an agent falls into two categories. One is to maximize the utility of temporary agent and improve the total throughput (from line 8 to line 14); the other is only to improve the total throughput (from line 17 to line 22) because, sometimes, the first purpose is difficult to achieve. Finally, the output of Algorithm 3 will be equal to 1 if deleting or adding an agent is successfully. Otherwise, it will return 0 .

Some notations and definitions in Algorithm 3 are as follow:


agent $_{1} \ldots$ agent $_{i} . .$. agent $\left._{n_{a}-1}\right\}$.Here, agent ${ }_{i}$ represents the $i^{\text {th }}$ agent in multi-agent system and $n_{a}$ is the total number of agents in current system.

- $s^{g}$ represents whether deleting or adding an agent is successful. If deleting or adding an agent is successful, $s^{g}$ will equal to1. Otherwise, $s^{g}$ will equal to 0 .

- $\operatorname{Obj}($ delete,$k)$ represents the total lack of QoS which is calculated under the condition that the $k^{\text {th }}$ agent is deleted.

- $\operatorname{Obj}(a d d,(s, f))$ represents the total lack of QoS which is calculated under the condition that the system add a new agent whose position is $s$ and frequency is $f$, respectively.

- agent $t^{t}$ represents temporary agent. $s^{t}, p^{t}, h^{t}, f^{t}$ represent its position, emitted power, azimuth and frequency, respectively.

- $U_{t}(s, f)$ represents the utility achieved by temporary agent if it moves to the position $s$ and its frequency is $f$.

Algorithm 3. The process of global control

Global_control(Agent)

1. $s^{g}=0$

2. $k_{\min }=\left\{k \mid \min \{\operatorname{Obj}(\right.$ delete,$\left.k)\}, k=0,1 \ldots n_{a}-1\right\}$

3. If $O b j\left(\right.$ delete,$\left.k_{\text {min }}\right)<o b j$

4. Agent $=$ Agent $\left\{\right.$ agent $\left._{k_{\text {min }}}\right\} \quad / /$ the system delete agent $_{k_{\min }}$

5. $n_{a}=n_{a}-1, s^{g}=1$

6. End if

7. If $s^{g}$ is equal to 0

8. Randomly generate $h^{t}, p^{t}$ for agent 
9. $\left(s_{\text {max }} f_{\text {max }}\right)=\left\{(s, f) \mid \max \left\{U_{t}(s, f)\right\}, s \in S^{e}, f \in F\right\}$

10. If $\operatorname{Obj}\left(a d d,\left(s_{\max }, f_{\text {max }}\right)\right)<o b j$

11. $s^{t}=s_{\max }, f^{t}=f_{\max }$

12. Agent $=$ Agent $\cup\left\{\right.$ agent $\left.^{t}\right\} / /$ agent $^{t}$ join the current system

13. $n_{a}=n_{a}+1, s^{g}=1$

14. End if

15. End if

16. If $s^{g}$ is equal to 0

17. $\left(s_{\text {min }}, f_{\text {min }}\right)=\left\{(s, f) \mid \min \{\operatorname{Obj}(\operatorname{add},(s, f))\}, s \in S^{e}, f \in F\right\}$

18. If $\operatorname{Obj}\left(a d d,\left(s_{\min }, f_{\min }\right)\right)<o b j$

19. $s^{t}=s_{\text {min }}, f^{t}=f_{\text {min }}$

20. Agent $=$ Agent $\cup\left\{\right.$ agent $\left.{ }^{t}\right\}$

21. $n_{a}=n_{a}+1, s^{g}=1$

22. End if

23. End if

24. Return $s^{g}$

Improved multi-agent optimization algorithm. Given the pseudo code of cooperative game and global control mentioned above, the improved multi-agent optimization algorithm is presented in Algorithm 4.

In the beginning of algorithm, there's only one agent (line 1 and line 2) generated in system. Through the system control (line 13), the number of agent can increase or the redundant agent may be deleted. Once the number of agents change successfully, the numbers of agents in current system will be rearranged according to the time when they are generated in the system. For example, if there exist three agents agent, agent $_{1}$,agent $_{2}\left(\right.$ agent $_{0}$ joined the system earliest while agent ${ }_{2}$ joined the system at the latest) and agent $t_{0}$ is deleted, then the subscript of agent ${ }_{1}$ will be equal to 0 (agent $_{1} \rightarrow$ agent $\left._{0}\right)$ and the subscript of agent $_{2}$ will be equal to $1\left(\right.$ agent $_{2} \rightarrow$ agent $\left._{1}\right)$.

The trigger condition of system control is that the actions of all agents are limited in cooperative game, which is demonstrated in line 12 of Algorithm 4 . In the line 12, $n_{a}$ is the number of agents in current system and $q$ represents the cumulative number of agents taking actions unsuccessfully (line 7). $q$ will be set to zero if one of the following two cases happens: one is that an agent does an action successfully (line 9); the other is that the total number of agents has changed (line 17).

Besides, in normal circumstances, the next agent will get ready to play game to maximize its utility when the last agent ends the game (line 11). However, when all agents can't do any action and the system changes the number of agents successfully, the next turn of cooperative game will start from the agent with number 0 (line 19).

In the end, the termination of the algorithm is that the total lack of QoS is equal to 0 (line 4 ) or the current system is unable to make any control to add a new agent or delete a redundant agent (line 15).

Algorithm 4. Improved multi-agent optimization algorithm

1. Randomly initialize agent $_{0}$ 
2. $n_{a}=1 \quad / /$ At first, the number of agents is only 1

3. $q=0, i=0$

4. While the total lack of QoS is not equal to 0

5. $p=$ Cooperative_game $(i)$

6. If $p$ is equal to 0

7. $q++/ /$ agent $i$ fail to take an action

8. Else

9. $q=0 \quad / /$ agent $i$ take an action successfully

10. End if

11. $i=(i+1) \bmod n_{a} / / \bmod$ is a modulo operation

12. If $q \geq n_{a} \quad / /$ judge whether the condition of system control is triggered

13. $p=$ Global_control(Agent)

14. If $p$ is equal to 0

15. break

16. Else

17. $q=0$ //The number of agents changes successfully

18. Rearrange the numbers of agents according to the time when they joined in the system

19. $i=0 \quad / /$ The next turn of cooperative game will start from agent $_{0}$

20. End if

21. End if

22. End while

23. Return Agent

\section{$4 \quad$ Experiment and result analysis}

\subsection{Experimental environment}

The complexity of solution space is not only related to the size of solution space, but also to the distribution and quantity of the near-optimal solution in the solution space. That means the problem with smaller solution space is not simpler than the problem with larger solution space. Therefore, two experimental scenarios are selected to verify the feasibility of the algorithm: One is a medium scale experimental scene with 252 candidate sites (described by the Figure 2) while the other is a large scale experimental scene only with 94 candidate sites (described by the Figure 3 ).

The test bed of medium scale experimental scene is composed of a three-floor building. Each floor size is $46 \mathrm{~m} \mathrm{x} 46 \mathrm{~m}$ ( $2116 \mathrm{MPs}$ for each floor). 88 candidate sites at 1 st floor, 77 candidate sites at 2 nd floor and 87 candidate sites at 3 rd floor. AP parameter settings are 1 type of AP with a unidirectional pattern, 1 type of power and 13 available frequencies under the $802.11 \mathrm{~b}$ standard. For the communication area, 100 users are uniformly distributed on each service zone (each floor) and each user demand is about $500 \mathrm{kbps}$ real bit rate. Consequently, the global demand for the whole building is $150000 \mathrm{kbps}$. 


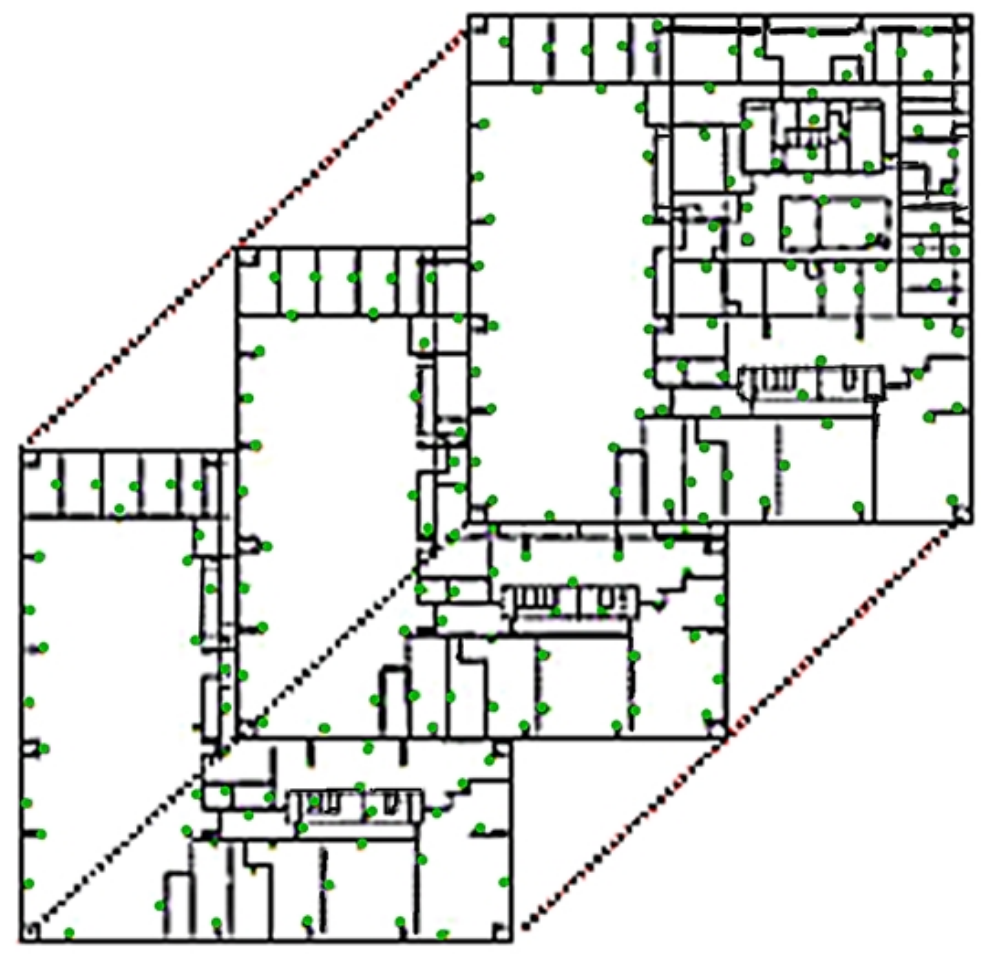

Fig. 2. The medium scale experimental scene

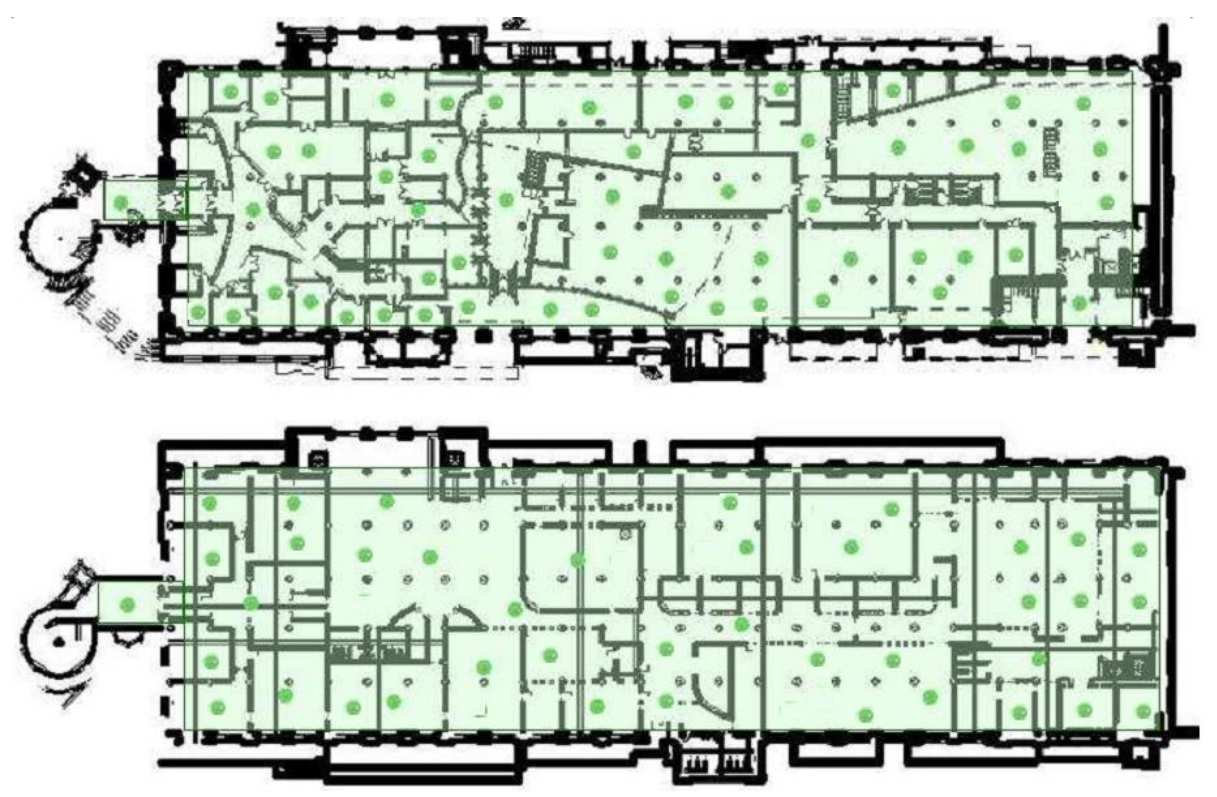

Fig. 3. The large scale experimental scene 


\subsection{Comparisons of two algorithms}

In order to prove the feasibility of our algorithm in low density of candidate sites, comparisons between our algorithm and the algorithm in Alan Mc Gibney's research work [12] will be presented. The results of these two algorithms are compared from two aspects. One is the best-run results of the two algorithms at different CPU times, which are illustrated in Figure 4 and Figure 5. The other is the minimum total lack of QoS that can be found in different cases of not exceeding a certain number of AP before both the algorithms converge, which are shown at Table 3 and Table 4, providing more clear choices for WLAN planner.

Performance of algorithms without limitation of APs. The curves in Figure 4 represent the convergence of the minimum total lack of QoS at medium scale scene. The green one illustrates the performance of our algorithm, and the blue one indicates the Alan Mc Gibney's. At early stage of search process (running time less than 28970s), Alan Mc Gibney's algorithm converges more rapidly than our algorithm for the reason that his algorithm does not take the relationship of channels among different agents into consideration when an agent moves to another new position. However, when these two algorithms achieve the state of convergence, the minimum total lack of QoS of our algorithm(790kb/s) is lower than Alan's $(1205 \mathrm{~kb} / \mathrm{s})$. What's more, the final convergence time spent by our algorithm is also shorter and the number of APs is also less (38 APs needed in our algorithm, but 79 APs in Alan's). It is because that the relationship of channels and positions of APs are considered synthetically in our algorithm which improve the efficiency of search in the solution space.

The test bed of large scale experimental scene is composed of a two-floor building. Each floor size is $120 \mathrm{~m} \times 40 \mathrm{~m}$ ( $4800 \mathrm{MPs}$ for each floor). 54 candidate sites at $1 \mathrm{st}$ floor and 40 candidate sites at 2 nd floor. The AP parameter settings are the same as the test bed of medium scale experimental scene. For the communication area, 400 users are uniformly distributed on each TP and each user demand is about $500 \mathrm{kbps}$ real bit rate. Consequently, the global demand for the whole building is $200000 \mathrm{kbps}$.

The curves in Figure 5 show the convergence of the minimum total lack of QoS at large scale scene. The green one is the performance of our algorithm, and the blue one represents the Alan Mc Gibney's. When running time reaches to 14870 s, the minimum total lack of QoS of Alan's algorithm falls into local minimum with 90 APs, while our algorithm reaching local minimum only needs 59 APs. The results indicate that using the relationship of field strength and channel can improve search efficiency of algorithm in solution space. Furthermore, the possibility of our algorithm to jump out of local minimum can be increased thanks to the whole adjustment of system. 


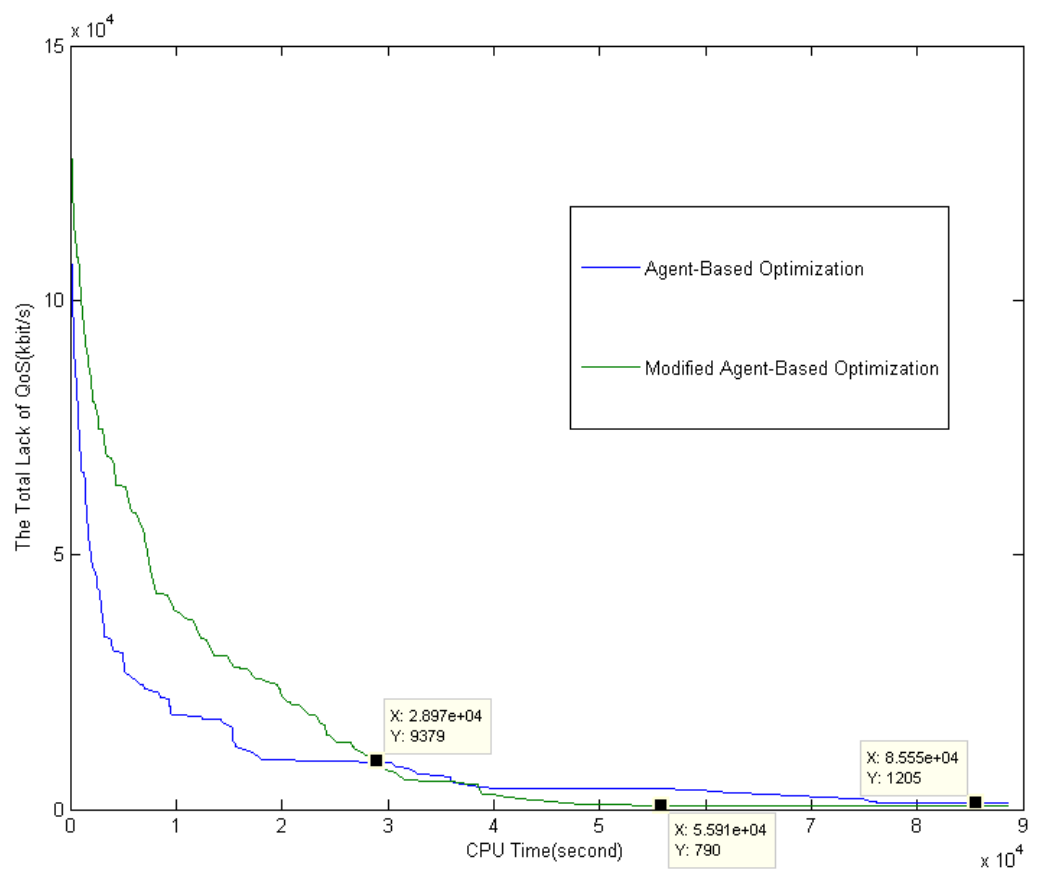

Fig. 4. The minimum total lack of QoS at medium scale scene



Fig. 5. The minimum total lack of QoS at large scale scene 
Performance of algorithms with limitation of APs. Both tables below support the conclusion that our algorithm is much more effective than Alan's algorithm. For instance, under the condition that the number of APs does not exceed to 45, the minimum total lack of QoS of our algorithm is only $790 \mathrm{~kb} / \mathrm{s}$ with 38 APs in Table 3, but Alan's algorithm reaches to $9836 \mathrm{~kb} / \mathrm{s}$ with 44 APs. What's more, since Alan's algorithm is more sensitive to the quantity of candidate sites, the superiority of our algorithm is more obvious in the case of a small number of APs.

Table 3. The minimum total lack of QoS at medium scale scene

\begin{tabular}{|c|c|c|c|c|}
\hline $\begin{array}{c}\text { Limited max } \\
\text { AP number }\end{array}$ & $\begin{array}{c}\text { The minimum total } \\
\text { lack of QoS in our } \\
\text { algorithm }\end{array}$ & $\begin{array}{c}\text { The minimum total lack } \\
\text { of QoS in Alan's algo- } \\
\text { rithm }\end{array}$ & $\begin{array}{c}\text { AP number in } \\
\text { our algorithm }\end{array}$ & $\begin{array}{c}\text { AP number in } \\
\text { Alan s algorithm }\end{array}$ \\
\hline 30 & $2839 \mathrm{~kb} / \mathrm{s}$ & $27082 \mathrm{~kb} / \mathrm{s}$ & 30 & 29 \\
\hline 35 & $955 \mathrm{~kb} / \mathrm{s}$ & $18537 \mathrm{~kb} / \mathrm{s}$ & 35 & 35 \\
\hline 40 & $790 \mathrm{~kb} / \mathrm{s}$ & $17044 \mathrm{~kb} / \mathrm{s}$ & 38 & 40 \\
\hline 45 & $790 \mathrm{~kb} / \mathrm{s}$ & $9836 \mathrm{~kb} / \mathrm{s}$ & 38 & 44 \\
\hline
\end{tabular}

Table 4. The minimum total lack of QoS at large scale scene

\begin{tabular}{|c|c|c|c|c|}
\hline $\begin{array}{c}\text { Limited max } \\
\text { AP number }\end{array}$ & $\begin{array}{c}\text { The minimum total } \\
\text { lack of QoS in our } \\
\text { algorithm }\end{array}$ & $\begin{array}{c}\text { The minimum total lack } \\
\text { of QoS in Alan's algo- } \\
\text { rithm }\end{array}$ & $\begin{array}{c}\text { AP number in } \\
\text { our algorithm }\end{array}$ & $\begin{array}{c}\text { AP number in } \\
\text { Alan's algorithm }\end{array}$ \\
\hline 30 & $38848 \mathrm{~kb} / \mathrm{s}$ & $48470 \mathrm{~kb} / \mathrm{s}$ & 30 & 30 \\
\hline 35 & $19823 \mathrm{~kb} / \mathrm{s}$ & $39138 \mathrm{~kb} / \mathrm{s}$ & 35 & 35 \\
\hline 40 & $8881 \mathrm{~kb} / \mathrm{s}$ & $32828 \mathrm{~kb} / \mathrm{s}$ & 40 & 40 \\
\hline 45 & $2275 \mathrm{~kb} / \mathrm{s}$ & $22941 \mathrm{~kb} / \mathrm{s}$ & 45 & 43 \\
\hline 50 & $1303 \mathrm{~kb} / \mathrm{s}$ & $20126 \mathrm{~kb} / \mathrm{s}$ & 50 & 50 \\
\hline 55 & $988 \mathrm{~kb} / \mathrm{s}$ & $19476 \mathrm{~kb} / \mathrm{s}$ & 55 & 51 \\
\hline
\end{tabular}

\section{Conclusion and Perspectives}

\subsection{Conclusion}

In this paper, a number of optimization methodologies applied to a large-scale WLAN design were investigated in previous work and some improvements for a multi-agent algorithm to optimize the practical engineering project of WLAN deployment with low density candidate sites scenarios are proposed. The contributions in our algorithm are as follows:

Firstly, the relationship of channel and field strength is considered synthetically, which can increase the diversity of search trajectory. Secondly, the global control of multi-agent system can help the search jump out of local optimum. Thirdly, our algorithm can increase the depth and breadth of search in current solution space by raising the barrier to add a new agent. The results of experiments have highlighted the better performance of our algorithm. 


\section{$5.2 \quad$ Perspectives}

With the number of candidate sites increasing, an agent can gain plenty of opportunities to adjust its position very slightly, weakening the integrated relationship between frequency and field strength and increasing the program running time. For example, in this paper, the running time in the medium scale scene is twice than that in the large scale scene. Therefore, our future work will focus on improving the algorithm efficiency by optimizing the action strategy of agents and balancing the search time and the result of optimization.

\section{Acknowledgment}

This work was supported by Zhejiang Provincial Natural Science Foundation of China (no.LQ14F010001, LY16F10014, LQ15F020004 LY18F020025), Key Scientific and Technological Projects of Ningbo (no. 2014B92001), Ningbo Natural Science Foundation (no.2017A610109, 2015A610132).

\section{$7 \quad$ References}

[1] Youngseok Lee, Kyoungae Kim and Yanghee Choi, "Optimization of AP placement and channel assignment in wireless LANs," 27th Annual IEEE Conference on Local Computer Networks, 2002. Proceedings. LCN 2002., 2002, pp. 831-836.

[2] E. Amaldi, A. Capone, M. Cesana, F. Malucelli and F. Palazzo, "WLAN coverage planning: optimization models and algorithms," 2004 IEEE 59th Vehicular Technology Conference. VTC 2004-Spring (IEEE Cat. No.04CH37514), 2004, pp. 2219-2223 Vol.4.

[3] L. Nagy and L. Farkas, "Indoor base station location optimization using genetic algorithms," 11th IEEE International Symposium on Personal Indoor and Mobile Radio Communications. PIMRC 2000. Proceedings (Cat. No.00TH8525), London, 2000, pp. 843-846 vol.2.

[4] Y. Zheng, O. Baala and A. Caminada, "Optimization model for an Indoor WLAN-based Positioning System," 2010 International Conference on Indoor Positioning and Indoor Navigation, Zurich, 2010, pp. 1-7. https://doi.org/10.1109/IPIN.2010.5648064

[5] B. Krishnamachari and S. B. Wicker, "Optimization of fixed network design in cellular systems using local search algorithms," Vehicular Technology Conference Fall 2000. IEEE VTS Fall VTC2000. 52nd Vehicular Technology Conference (Cat. No.00CH37152), Boston, MA, 2000, pp. 1632-1638 vol.4. https://doi.org/10.1109/VETECF.2000.886104

[6] S. Hurley, "Planning effective cellular mobile radio networks," in IEEE Transactions on Vehicular Technology, vol. 51, no. 2, pp. 243-253, Mar 2002. https://doi.org/10.1109/ 25.994802

[7] Pierre Hansen, Nenad Mladenović, "Variable neighborhood search: Principles and applications," European Journal of Operational Research, 130.3(2001):449-467. https://doi.org/10.1016/S0377-2217(00)00100-4

[8] K. F. Man, K. S. Tang, and S. Kwong.(1999). Genetic Algorithms. Springer London. https://doi.org/10.1007/978-1-4471-0577-0

[9] Dongmei Yan, Jinkuan Wang, Li Liu and Jing Gao, "Target tracking based on cluster and game theory in wireless sensor network," IET 2nd International Conference on Wireless, Mobile and Multimedia Networks, Beijing, China, 2008, pp. 45-48. 
[10] Ines Sghir, Jin-Kao Hao, Ines Ben Jaafar, and Khaled Ghédira, "A multi-agent based optimization method applied to the quadratic assignment problem," Expert Systems with Applications: An International Journal, vol. 42, pp. 9252-9262, December 2015. https://doi.org/10.1016/j.eswa.2015.07.070

[11] K. Luo and W. Shi, "Distributed coordination method of microgrid economic operation optimization based on multi-agent system," 2014 International Conference on Power System Technology, Chengdu, 2014, pp. 3135-3140.

[12] Alan Mc Gibney, Martin Klepal and Dirk Pesch, "Agent-Based Optimization for Large Scale WLAN Design," in IEEE Transactions on Evolutionary Computation, vol. 15, no. 4, pp. 470-486, Aug. 2011. https://doi.org/10.1109/TEVC.2010.2064324

[13] Alan Mc Gibney, Martin Klepal and Dirk Pesch, "WLAN Design: A Distributed Approach," VTC Spring 2008 - IEEE Vehicular Technology Conference, Singapore, 2008, pp. 371-375.

[14] E. Semsar-Kazerooni and K. Khorasani, "A game theory approach to multi-agent team cooperation," 2009 American Control Conference, St. Louis, MO, 2009, pp. 4512-4518. https://doi.org/10.1109/ACC.2009.5160273

[15] Y. Prudent and A. Ennaji, "An incremental growing neural gas learns topologies," Proceedings. 2005 IEEE International Joint Conference on Neural Networks, 2005, pp. 12111216 vol. 2. https://doi.org/10.1109/IJCNN.2005.1556026

[16] Alexandre Gondran, Alexandre Caminada, Julien Fondrevelle, and Oumaya Baala, "Wireless LAN planning: a didactical model to optimise the cost and effective payback," International Journal of Mobile Network Design \&Innovation, vol. 2, pp. 13-25, May 2007. https://doi.org/10.1504/IJMNDI.2007.013800

[17] Katia Jaffres-Runser, Jean-Marie Gorce, and Stephane Ubeda, "Mono- and multiobjective formulations for the indoor wireless LAN planning problem," Computers \& Operations Research, vol.35, pp. 3885-3901, December 2008. https://doi.org/10.1016/j.cor.2007. 02.011

[18] Jangeun Jun, P. Peddabachagari and M. Sichitiu, "Theoretical maximum throughput of IEEE 802.11 and its applications," Second IEEE International Symposium on Network Computing and Applications, 2003, Cambridge, MA, USA, 2003, pp. 249-256.

[19] George Mavrotas.(2009). Effective implementation of the $\varepsilon$-constraint method in MultiObjective Mathematical Programming problems. Applied Mathematics and Computation: vol. 213, pp. 455-465, July, 2009. https://doi.org/10.1016/j.amc.2009.03.037

[20] Hou D, "Models, theory and applications in cooperative game theory," Enschede: Universiteit Twente, 2013. https://doi.org/10.3990/1.9789036500050

\section{Authors}

You Zheng was born in 1981, Ph.D. He is Post-Doctor with Wuhan University of Technology. His current research interests focus on modeling and optimization for telecommunication system.

Xiaohuo Xu is with Ningbo University of Technology, Ningbo, China (e-mail: huosai008@qq.com).

Tailong Shi is with Ningbo University of Technology, Ningbo, China.

Dandan Fang is Post-Doctor with Wuhan University of Technology. Her current research interests focus on artificial intelligence.

Peng An is the associate professor with Ningbo University of Technology. His current research interests focus on embedded system. 